IPNO/TH 97-06

\title{
The infinite mass limit of the two-particle Green's function in QED
}

\author{
H. Jallouli円 and H. Sazdjian巴 \\ Division de Physique Théoriqu€, Institut de Physique Nucléaire, \\ Université Paris XI, \\ F-91406 Orsay Cedex, France
}

\begin{abstract}
The behavior of the two-particle Green's function in QED is analyzed in the limit when one of the particles becomes infinitely massive. It is found that the dependences of the Green's function on the relative times of the ingoing and outgoing particles factorize and that the bound state spectrum is the same as that of the Dirac equation with the static potential created by the heavy particle. The Bethe-Salpeter wave function is also determined in terms of the Dirac wave function. The present result excludes the existence, in the above limit, of abnormal solutions due to relative time excitations as predicted by the Bethe-Salpeter equation in the ladder approximation.
\end{abstract}

PACS numbers: 11.10.St, 12.20.Ds.

Keywords: Relativistic bound states, Bethe-Salpeter equation, Quantum Electrodynamics.

\footnotetext{
${ }^{1}$ E-mail: jallouli@ipno.in2p3.fr .

${ }^{2}$ E-mail: sazdjian@ipno.in2p3.fr .

${ }^{\ddagger}$ Unité de Recherche des Universités Paris 11 et Paris 6 associée au CNRS.
} 


\section{Introduction}

It is generally admitted that the infinite mass limit of the two-particle Green's function in QED (or in similar abelian theories) yields for the lighter particle the Dirac equation in the presence of the static potential created by the heavy one. The corresponding derivations are essentially based on mass-shell [1, 2, 3] or quasi-mass-shell [4, 6, 6] conditions. On the other hand, it is known that the Bethe-Salpeter equation in the ladder approximation leads to the appearance of "abnormal" solutions in the spectrum, due to the relative time excitations [7, 8, 9, 10]. Taking the infinite mass limit (for one of the particles) does not remove from the spectrum the above solutions $[8]$ and therefore the corresponding limiting equation is not equivalent to the Dirac equation with a static potential. It is noteworthy to mention that in the static limit, when both particle masses go to infinity, the abnormal solutions still persist in the ladder approximation [11].

In a recent work, we showed that in the static limit the two-particle Green's function can exactly be calculated and the corresponding bound state spectrum does not display any abnormal solution [12]. This result strongly suggests that the appearance of the abnormal solutions is rather a consequence of the ladder approximation (with covariant propagators) of the Bethe-Salpeter equation. It is then natural to expect that also the same conclusion might hold in the one-particle limit.

The purpose of the present article is to study the (one-particle) infinite mass limit of the two-particle Green's function without making any mass-shell or quasi-mass-shell assumptions on the heavy particle and therefore not constraining the relative time evolution of the system. We shall show that the dependences of the Green's function on the relative times of the ingoing and outgoing particles factorize and that its exact spectrum in the above limit (in the absence of radiative corrections) is given by that of the Dirac equation of the lighter particle in the presence of the static potential. The relative time variables, without being absent from the expressions of the Green's function and of the Bethe-Salpeter wave function do not induce energy excitations, but rather ensure by their presence the correct spectral properties as dictated by quantum field theory [7, 9]. This result also also allows the determination of the Bethe-Salpeter wave function in terms of the Dirac wave function.

While in the above calculations radiative corrections of the lighter particle are neglected (or partly taken into account in covariant gauges), this approximation does not seem crucial for the derivation of the factorization property of the Green's function in the relative time variables, since this feature is rather related to the heavy particle properties. By appropriately generalizing the static potential so as to include the full radiative corrections of the lighter particle, one should still be able to prove the main qualitative results as described above. 
The QED bound state spectrum being gauge invariant [15, 16] it is actually sufficient to prove the above properties in one particular gauge. We shall choose the Coulomb gauge for a detailed derivation, but shall also briefly sketch the line of calculation with linear covariant gauges.

\section{The two-particle Green's function with an infinite mass}

Our starting point is the QED lagrangian density for two fermions with masses $m_{1}$ and $m_{2}$ and charges $e_{1}=e_{2}=-e$ :

$$
\begin{aligned}
\mathcal{L}=\bar{\psi}_{1}(i \gamma . \partial & \left.-m_{1}+e \gamma \cdot A\right) \psi_{1}+\bar{\psi}_{2}\left(i \gamma . \partial-m_{2}+e \gamma \cdot A\right) \psi_{2} \\
& -\frac{1}{4} F_{\mu \nu} F^{\mu \nu}+\mathcal{L}_{\} .\{.},
\end{aligned}
$$

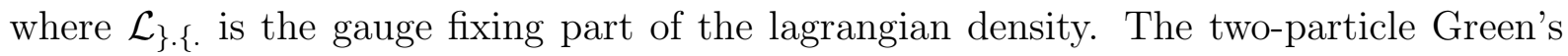
function is defined by the functional integral:

$$
\begin{aligned}
G\left(x_{1}, x_{2}, x_{1}^{\prime}, x_{2}^{\prime}\right) & \left.=\mathcal{N} \int \mathcal{D} \psi \mathcal{D} \bar{\psi} \mathcal{D} \mathcal{A} \psi_{\infty}\left(\S_{\infty}\right) \psi_{\epsilon}\left(\S_{\epsilon}^{\prime}\right) \bar{\psi}_{\epsilon}\left(\S_{\epsilon}\right) \bar{\psi}_{\infty}\left(\S_{\infty}^{\prime}\right)\right\rceil^{\gamma \mathcal{S}(\psi, \bar{\psi}, \mathcal{A})} \\
& \equiv<\psi_{1}\left(x_{1}\right) \psi_{2}\left(x_{2}^{\prime}\right) \bar{\psi}_{2}\left(x_{2}\right) \bar{\psi}_{1}\left(x_{1}^{\prime}\right) e^{i S(\psi, \bar{\psi}, A)}>
\end{aligned}
$$

where $S=\int d^{4} x \mathcal{L}(\S)$ is the action. We shall be interested by the bound state spectrum of the system fermion 1-antifermion 2. We consider particle 2 as the heavy one and take the limit of large $m_{2}$. This limit can be studied by treating perturbatively the (covariant) kinetic energy term of particle 2. Such a situation has often been met in heavy quark effective field theories [13]. A way of implementing this limit is to make successive transformations on the fermion 2 field, the first of which being [14]:

$$
\psi_{2} \rightarrow \exp \left(\left(i \gamma_{i} \partial^{i}+e \gamma_{i} A^{i}\right) /\left(2 m_{2}\right)\right) \psi_{2}, \quad \bar{\psi}_{2} \rightarrow \bar{\psi}_{2} \exp \left(\left(-i \gamma_{i} \overleftarrow{\partial^{i}}+e \gamma_{i} A^{i}\right) /\left(2 m_{2}\right)\right)
$$

This transformation has been shown to have unit jacobian [14. It eliminates at order $m_{2}^{0}$ the (covariant) kinetic energy part of the fermion 2 lagrangian density and the corresponding free propagator becomes the static one:

$$
\begin{aligned}
G_{20}\left(x_{2}^{\prime}-x_{2}\right)= & \frac{1}{2}\left(1+\gamma_{0}\right) e^{-i m_{2}\left(x_{2}^{\prime 0}-x_{2}^{0}\right)} \theta\left(x_{2}^{\prime 0}-x_{2}^{0}\right) \delta^{3}\left(\mathbf{x}_{\mathbf{2}}^{\prime}-\mathbf{x}_{\mathbf{2}}\right) \\
& +\frac{1}{2}\left(1-\gamma_{0}\right) e^{-i m_{2}\left(x_{2}^{0}-x_{2}^{\prime 0}\right)} \theta\left(x_{2}^{0}-x_{2}^{\prime 0}\right) \delta^{3}\left(\mathbf{x}_{\mathbf{2}}-\mathbf{x}_{\mathbf{2}}^{\prime}\right)
\end{aligned}
$$

The antifermion contribution is selected when $x_{2}^{0}>x_{2}^{\prime 0}$ and we shall henceforth consider only this case $\left(\gamma_{0}\right.$ has then the eigenvalue -1$)$. 
Integration of the functional integral (2) with respect to the photon field amounts to replacing the action $S$ by an effective one $\bar{S}=\int d^{4} x d^{4} y \overline{\mathcal{L}}(x, y)$, with

$$
\begin{aligned}
\overline{\mathcal{L}}(x, y)= & \left(\bar{\psi}_{1}\left(i \gamma . \partial-m_{1}\right) \psi_{1}+\psi_{2}\left(i \gamma_{0} \partial^{0}-m_{2}\right) \psi_{2}\right)_{x} \delta^{4}(x-y) \\
& +\frac{i e^{2}}{2}\left(j_{1 \mu}(x)+j_{2 \mu}(x)\right) D^{\mu \nu}(x-y)\left(j_{1 \nu}(y)+j_{2 \nu}(y)\right),
\end{aligned}
$$

where $j_{a \mu} \equiv \bar{\psi}_{a} \gamma_{\mu} \psi_{a}, a=1,2$, is the current of particle $a$, with $j_{2 \mu}=g_{\mu}{ }^{0} j_{20}$, and $D_{\mu \nu}$ is the photon propagator. We shall neglect in the following tha radiative corrections and therefore shall maintain in the interaction part of $\overline{\mathcal{L}}$ the crossed terms $j_{1} D j_{2}+j_{2} D j_{1}$ only. (Actually, the radiative corrections relative to the heavy particle 2 can explicitly be calculated [12 and the latter approximation concerns rather the lighter particle 1.)

Use of Wick's theorem with respect to particle 2 together with formula (1) and condition $x_{2}^{0}>x_{2}^{\prime 0}$ yields for the Green's function (2) the expression:

$$
\begin{aligned}
G\left(x_{1}, x_{2}, x_{1}^{\prime}, x_{2}^{\prime}\right) & =G_{20}\left(x_{2}^{\prime}-x_{2}\right)<\psi_{1}\left(x_{1}\right) \bar{\psi}_{1}\left(x_{1}^{\prime}\right) \\
& \times \exp \left\{i \int d^{4} y_{1} \bar{\psi}_{1}\left(y_{1}\right)\left(i \gamma_{1} . \partial_{1}-m_{1}-i e^{2} \gamma_{1}^{\mu} \widetilde{A}_{\mu}\left(y_{1}, x_{2}, x_{2}^{\prime}\right)\right) \psi_{1}\left(y_{1}\right)\right\}> \\
\widetilde{A}_{\mu}\left(y_{1}, x_{2}, x_{2}^{\prime}\right) & =\int_{x_{2}^{\prime 0}}^{x_{2}^{0}} d y_{2}^{0} D_{\mu 0}\left(y_{1}^{0}-y_{2}^{0}, \mathbf{y}_{\mathbf{1}}-\mathbf{x}_{\mathbf{2}}\right) .
\end{aligned}
$$




\section{Coulomb gauge calculation of the energy spectrum}

In the Coulomb gauge, $\widetilde{A}_{\mu}$ is:

$$
\widetilde{A}_{\mu}^{(C)}\left(y_{1}, x_{2}, x_{2}^{\prime}\right)=i g_{\mu 0} \frac{1}{4 \pi} \frac{1}{\left|\mathbf{y}_{\mathbf{1}}-\mathbf{x}_{\mathbf{2}}\right|} \theta\left(x_{2}^{0}-y_{1}^{0}\right) \theta\left(y_{1}-x_{2}^{\prime 0}\right) .
$$

The Green's function (7) can be evaluated by considering the differential equations it satisfies. These are:

$$
\begin{array}{r}
{\left[i \gamma_{10} \partial_{10}+i \gamma_{1}^{i} \partial_{1 i}-m_{1}-\gamma_{10} V(r) \theta\left(x_{2}^{0}-x_{1}^{0}\right) \theta\left(x_{1}^{0}-x_{2}^{\prime 0}\right] G\left(x_{1}, x_{2}, x_{1}^{\prime}, x_{2}^{\prime}\right)\right.} \\
=G_{20}\left(x_{2}^{\prime}-x_{2}\right) i \delta^{4}\left(x_{1}-x_{1}^{\prime}\right) \\
G\left(x_{1}, x_{2}, x_{1}^{\prime}, x_{2}^{\prime}\right)\left[-i \gamma_{10} \overleftarrow{\partial_{10}^{\prime}}-i \gamma_{1}^{i} \overleftarrow{\partial_{1 i}^{\prime}}-m_{1}-\gamma_{10} V\left(r^{\prime}\right) \theta\left(x_{2}^{0}-x_{1}^{\prime 0}\right) \theta\left(x_{1}^{\prime 0}-x_{2}^{\prime 0}\right)\right] \\
=G_{20}\left(x_{2}^{\prime}-x_{2}\right) i \delta^{4}\left(x_{1}-x_{1}^{\prime}\right)
\end{array}
$$

where

$$
V(r)=-\frac{\alpha}{r}, \quad \alpha=\frac{e^{2}}{4 \pi}, \quad r=\left|\mathbf{x}_{\mathbf{1}}-\mathbf{x}_{\mathbf{2}}\right|, \quad \mathbf{r}^{\prime}=\left|\mathbf{x}_{\mathbf{1}}^{\prime}-\mathbf{x}_{\mathbf{2}}^{\prime}\right| .
$$

Before solving equations (2)-(3) we shall introduce a particular representation for the one-particle Green's function which will be useful throughout the resolution procedure. Let us first consider the free Green's function of particle 1. Defining in momentum space the functions

$$
h_{0}\left(\mathbf{p}_{\mathbf{1}}\right)=\gamma_{\mathbf{0}}\left(\gamma_{\mathbf{i}} \mathbf{p}_{\mathbf{1}}^{\mathbf{i}}+\mathbf{m}_{\mathbf{1}}\right), \quad \sqrt{\mathbf{h}_{\mathbf{0}}^{2}}=\sqrt{\mathbf{m}_{1}^{2}+\mathbf{p}_{\mathbf{1}}^{2}},
$$

and the projectors

$$
\Lambda_{0 \pm}=\frac{1}{2}\left(1 \pm \frac{h_{0}}{\sqrt{h_{0}^{2}}}\right)
$$

one can write the free Green's function in the form:

$$
G_{10}=\frac{i}{p_{10}-h_{0}+i \epsilon} \Lambda_{0+} \gamma_{0}+\frac{i}{p_{10}-h_{0}-i \epsilon} \Lambda_{0-} \gamma_{0}
$$

It takes in $x$-space the form:

$$
G_{10}\left(x_{1}-x_{1}^{\prime}\right)=e^{-i x_{1}^{0} h_{0}} \frac{1}{2}\left(\epsilon\left(x_{1}^{0}-x_{1}^{\prime 0}\right)+\frac{h_{0}}{\sqrt{h_{0}^{2}}}\right) \delta^{3}\left(\mathbf{x}_{\mathbf{1}}-\mathbf{x}_{1}^{\prime}\right) \gamma_{\mathbf{0}} \mathbf{e}^{\mathbf{i} \mathbf{x}_{\mathbf{1}}^{\prime \mathbf{0}} \mathbf{h}_{\mathbf{o}}^{\prime}}
$$

where now $h_{0}$ and $h_{0}^{\prime}$ are the $x$-space expressions of the function $h_{0}\left(\mathbf{p}_{\mathbf{1}}\right)$ :

$$
h_{0}=-i \gamma_{0} \gamma^{i} \partial_{1 i}+m_{1} \gamma_{0}, \quad h_{0}^{\prime}=-i \gamma_{0} \gamma^{i} \overleftarrow{\partial_{1 i}^{\prime}}+m_{1} \gamma_{0}
$$

By replacing, through the closure relation, the delta-function above by a complete set of spinor wave functions and identifying the arguments of the exponential functions with 
the energy eigenvalues of these functions, one deduces that for $x_{1}^{0}>x_{1}^{\prime 0}$ they correspond to the positive energy solutions of the free Dirac equation and for $x_{1}^{0}<x_{1}^{\prime 0}$ to the the negative energy ones, thus exhausting the spectrum of states saturating the free Green's function.

The above construction can also be generalized to the case of the one-particle Green's function in the presence of a static vector potential $V(r)$. The Green's function then satisfies the differential equation

$$
\left(i \gamma_{1} . \partial_{1}-m_{1}-\gamma_{10} V(r)\right) G_{1}\left(x_{1}, x_{1}^{\prime}, \mathbf{x}_{2}\right)=\mathbf{i} \delta^{4}\left(\mathbf{x}_{1}-\mathbf{x}_{1}^{\prime}\right)
$$

and its conjugate one. Since $V$ is static, the formal solution of these equations can be obtained from expression (8) by a shift of the operators $h_{0}$ and $h_{0}^{\prime}$ by the factor $V$. Defining:

$$
h=-i \gamma_{0} \gamma^{i} \partial_{1 i}+m_{1} \gamma_{0}+V(r), \quad h^{\prime}=-i \gamma_{0} \gamma^{i} \overleftarrow{\partial_{1 i}^{\prime}}+m_{1} \gamma_{0}+V\left(r^{\prime}\right)
$$

one obtains:

$$
G_{1}\left(x_{1}, x_{1}^{\prime}, \mathbf{x}_{2}\right)=\mathbf{e}^{-\mathbf{i} \mathbf{x}_{1}^{0} \mathbf{h}} \frac{\mathbf{1}}{2}\left(\epsilon\left(\mathbf{x}_{1}^{0}-\mathbf{x}_{1}^{\prime \mathbf{0}}\right)+\frac{\mathbf{h}}{\sqrt{\mathbf{h}^{\mathbf{2}}}}\right) \delta^{3}\left(\mathbf{x}_{1}-\mathbf{x}_{1}^{\prime}\right) \gamma_{0} \mathbf{e}^{\mathbf{i} \mathbf{x}_{1}^{\prime 0} \mathbf{h}^{\prime}} .
$$

$\left[\sqrt{h^{2}}\right.$ is an appropriate generalization of $\sqrt{h_{0}^{2}}$. $]$ We also notice the following property of the operators $h$ and $h^{\prime}$ :

$$
h \delta^{3}\left(\mathbf{x}_{\mathbf{1}}-\mathbf{x}_{\mathbf{1}}^{\prime}\right) \gamma_{\mathbf{0}}=\delta^{\mathbf{3}}\left(\mathbf{x}_{\mathbf{1}}-\mathbf{x}_{\mathbf{1}}^{\prime}\right) \gamma_{\mathbf{0}} \mathbf{h}^{\prime}
$$

which allows us to convert, when necessary for symmetry reasons, the operators $h$ and $h^{\prime}$ into each other. Again the use of the closure relation through the delta-function allows us to identify the spectrum os states saturating the Green's function with the set of solutions of the Dirac equation in the presence of the static potential.

We are now in a position to construct the solutions of Eqs. (2)-(3)). We first rewrite them in the form:

$$
\begin{gathered}
{\left[i \partial_{10}-h+V\left(1-\theta\left(x_{2}^{0}-x_{1}^{0}\right) \theta\left(x_{1}-x_{2}^{\prime 0}\right)\right)\right] G=G_{20} i \delta^{4}\left(x_{1}-x_{1}^{\prime}\right) \gamma_{10}} \\
G\left[-i \overleftarrow{\partial_{10}^{\prime}}-h^{\prime}+V \mid\left(1-\theta\left(x_{2}^{0}-x_{1}^{\prime 0}\right) \theta\left(x_{1}^{\prime 0}-x_{2}^{\prime 0}\right)\right)\right]=G_{20} i \delta^{4}\left(x_{1}-x_{1}^{\prime}\right) \gamma_{10}
\end{gathered}
$$

After making the change of function $G=e^{-i x_{1}^{0} h} \widetilde{G} e^{i x_{1}^{\prime 0} h^{\prime}}$, one integrates for $\widetilde{G}$, by imposing the boundary condition that in the limit $x_{2}^{\prime 0} \rightarrow-\infty$ and $x_{2}^{0} \rightarrow \infty$, with $x_{1}^{\prime 0}$ and $x_{1}^{0}$ fixed, $G$ 
tends, up to multiplicative factors, to the static potential case solution (12), as is evident from Eqs. (2)-(3). One finds the solution:

$$
\begin{aligned}
G=G_{20} & \left.x_{2}^{\prime}-x_{2}\right) e^{-i x_{1}^{0} h} T \exp \left\{i \int_{x_{1}^{\prime 0}}^{x_{1}^{0}} d y_{1}^{0} e^{i y_{1}^{0} h} V(r)\left(1-\theta\left(x_{2}^{0}-y_{1}^{0}\right) \theta\left(y_{1}^{0}-x_{2}^{\prime 0}\right)\right) e^{-i y_{1}^{0} h}\right\} \\
& \times \frac{1}{2}\left(\epsilon\left(x_{1}^{0}-x_{1}^{\prime 0}\right)+\frac{h}{\sqrt{h^{2}}}\right) \delta^{3}\left(\mathbf{x}_{\mathbf{1}}-\mathbf{x}_{\mathbf{1}}^{\prime}\right) \gamma_{\mathbf{1 0}} \mathbf{e}^{\mathbf{i} \mathbf{x}_{\mathbf{1}}^{\prime \mathbf{0}} \mathbf{h}^{\prime}}
\end{aligned}
$$

where $T$ designates the chronological product. This expression can be further simplified for the situation that interests us. Sticking for definiteness to the case $x_{2}^{0}$ and $x_{1}^{0}$ greater than $x_{2}^{\prime 0}$ and $x_{1}^{\prime 0}$, one observes that the domain of integration $\left[x_{2}^{\prime 0}, x_{2}^{0}\right]$ does not contribute to the above integral. One therefore obtains two disjoint domains of integrations, which allow the factorization of the $T$-product. Using then the property (13), the expression of $G$ becomes:

$$
\begin{aligned}
G=G_{20} & \left.x_{2}^{\prime}-x_{2}\right) e^{-i x_{1}^{0} h} T \exp \left\{i \int_{x_{2}^{0}}^{x_{1}^{0}} d y_{1}^{0} e^{i y_{1}^{0} h} V(r)\left(1-\theta\left(x_{2}^{0}-y_{1}^{0}\right) \theta\left(y_{1}^{0}-x_{2}^{\prime 0}\right)\right) e^{-i y_{1}^{0} h}\right\} \\
& \times \frac{1}{2}\left(\epsilon\left(x_{1}^{0}-x_{1}^{\prime 0}\right)+\frac{h}{\sqrt{h^{2}}}\right) \delta^{3}\left(\mathbf{x}_{\mathbf{1}}-\mathbf{x}_{\mathbf{1}}^{\prime}\right) \gamma_{\mathbf{1 0}} \\
\times & T \exp \left\{i \int_{x_{1}^{\prime 0}}^{x_{2}^{\prime 0}} d y_{1}^{0} e^{i y_{1}^{0} h^{\prime}} V\left(r^{\prime}\right)\left(1-\theta\left(x_{2}^{0}-y_{1}^{0}\right) \theta\left(y_{1}^{0}-x_{2}^{\prime 0}\right)\right) e^{-i y_{1}^{0} h^{\prime}}\right\} .
\end{aligned}
$$

Introducing the relative variables $x=x_{1}-x_{2}$ and $x^{\prime}=x_{1}^{\prime}-x_{2}^{\prime}$, and making in the integrals appropriate changes of variables, and also using the property $\exp \left(e^{B} A e^{-B}\right)=e^{B} e^{A} e^{-B}$, one finally obtains for $G$ the expression:

$$
\begin{aligned}
G=G_{20} & \left(x_{2}^{\prime}-x_{2}\right) T \exp \left\{i \int_{0}^{x^{0}} d z^{0} e^{-i z^{0} h} V(r) \theta\left(x^{0}-z^{0}\right) e^{i z^{0} h}\right\} \\
& \times e^{-i x_{1}^{0} h} \frac{1}{2}\left(\epsilon\left(x_{1}^{0}-x_{1}^{\prime 0}\right)+\frac{h}{\sqrt{h^{2}}}\right) \delta^{3}\left(\mathbf{x}_{\mathbf{1}}-\mathbf{x}_{\mathbf{1}}^{\prime}\right) \gamma_{\mathbf{1 0}} \mathbf{e}^{\mathbf{i} \mathbf{x}_{\mathbf{1}}^{\prime 0} \mathbf{h}^{\prime}} \\
& \times T \exp \left\{-i \int_{0}^{x^{0}} d z^{0} e^{-i z^{0} h^{\prime}} V\left(r^{\prime}\right) \theta\left(z^{0}-x^{\prime 0}\right) e^{i z^{0} h^{\prime}}\right\} .
\end{aligned}
$$

Using for the delta-function the closure relation:

$$
\delta^{3}\left(\mathbf{x}_{\mathbf{1}}-\mathbf{x}_{\mathbf{1}}^{\prime}\right)=\sum_{\mathbf{n}} \psi_{\mathbf{n}}(\mathbf{x}) \bar{\psi}_{\mathbf{n}}\left(\mathbf{x}^{\prime}\right)
$$

[from the expression of $G_{20}$, Eq. (田), we have $\mathbf{x}_{\mathbf{2}}=\mathbf{x}_{\mathbf{2}}^{\prime}$ ], where the $\psi_{n}$ 's form a complete set of spinor wave functions, and comparing the resulting expression of $G$ with its cluster decomposition formula (for $x_{2}^{0}$ and $x_{1}^{0}$ greater than $x_{2}^{\prime 0}$ and $x_{1}^{\prime 0}$ ) [17]:

$$
G\left(x_{1}, x_{2}, x_{1}^{\prime}, x_{2}^{\prime}\right)=\sum_{n} \Phi_{n}\left(x_{1}, x_{2}\right) \bar{\Phi}_{n}\left(x_{1}^{\prime}, x_{2}^{\prime}\right)=\sum_{n} \phi_{n}(x) e^{-i P_{n} \cdot\left(X-X^{\prime}\right)} \bar{\phi}_{n}\left(x^{\prime}\right),
$$

where the $\Phi$ 's are generalized Bethe-Salpeter wave functions, one deduces the energy spectrum of the theory. [We have introduced the total momentum variable $P=p_{1}+p_{2}$ 
and the total coordinates $X=\left(x_{1}+x_{2}\right) / 2$ and $X^{\prime}$.] By identifying in the expression of $G$ the argument of the exponential functions containing the total coordinates $X^{0}$ and $X^{\prime 0}$ with the energy eigenvalues, one deduces that the energy spectrum is that of the positive energy solutions of the Dirac equation with the static potential $V$ (added by $m_{2}$ ):

$$
\left(i \gamma_{1} . \partial_{1}-m_{1}-\gamma_{10} V(r)\right) \psi\left(x_{1}^{0}, \mathbf{x}_{\mathbf{1}}-\mathbf{x}_{\mathbf{2}}\right)=\mathbf{0} .
$$

Repeating the above operations with values of $x_{2}^{0}$ and $x_{1}^{\prime 0}$ greater than $x_{2}^{\prime 0}$ and $x_{1}^{0}$, one recovers the negative energy solutions of Eq. (21). Therefore, the energy spectrum of the two-particle Green's function (2) in the limit $m_{2} \rightarrow \infty$ is given, up to the additive factor $m_{2}$, by the spectrum of eigenvalues of Eq. (21). This spectrum does not contain any abnormal solutions corresponding to the relative time excitations.

The previous procedure makes also possible the identification of the Bethe-Salpeter wave function in terms of the Dirac wave function $\psi$. Designating by $p_{10}$ the energy eigenvalue of the Dirac wave function, one has for the bound state wave functions (for which $\left.x_{1}^{0}>x_{1}^{\prime 0}\right)$ :

$$
\Phi\left(x_{1}, x_{2}\right)=e^{-i\left(m_{2} x_{2}^{0}+p_{10} x_{1}^{0}\right)} T \exp \left\{i \int_{0}^{x^{0}} d z^{0} e^{-i z^{0} h} V(r) \theta\left(x^{0}-z^{0}\right) e^{i z^{0} h}\right\} \psi(\mathbf{x}) .
$$

For $x^{0}<0$, the integral vanishes and one obtains:

$$
\Phi=e^{-i\left(m_{2} x_{2}^{0}+p_{10} x_{1}^{0}\right)} \psi(\mathbf{x})=\mathbf{e}^{-\mathbf{i}\left(\mathbf{m}_{1}+\mathbf{m}_{2}+\mathbf{E}\right) \mathbf{X}^{\mathbf{0}}} \mathbf{e}^{-\mathbf{i}\left(\mathbf{m}_{1}-\mathbf{m}_{\mathbf{2}}\right) \mathbf{x}^{\mathbf{0}} / \mathbf{2}} \mathbf{e}^{-\mathbf{i} \mathbf{E x} \mathbf{x}^{\mathbf{0}} / \mathbf{2}} \psi(\mathbf{x}), \quad \mathbf{x}^{\mathbf{0}}<\mathbf{0},
$$

where we have introduced the binding energy $E$ :

$$
p_{10}=m_{1}+E, \quad E<0 .
$$

For $x^{0}>0$, the $\theta$-function in the integral can be replaced by 1 , and using the relation [18]

$$
e^{(A+B) t} e^{-A t}=T \exp \left\{\int_{0}^{t} d t^{\prime} e^{A t^{\prime}} B e^{-A t^{\prime}}\right\},
$$

one finds:

$$
\begin{aligned}
\Phi & =e^{-i\left(m_{2}+p_{10}\right) x_{2}^{0}} e^{-i x^{0}(h-V)} \psi(\mathbf{x}) \\
& =e^{-i\left(m_{1}+m_{2}+E\right) X^{0}} e^{-i\left(m_{1}-m_{2}\right) x^{0} / 2} e^{i E x^{0} / 2} e^{-i x^{0}\left(h_{0}-m_{1}\right)} \psi(\mathbf{x}), \quad \mathbf{x}^{\mathbf{0}}>\mathbf{0} .
\end{aligned}
$$

In the bound state domain, the operator $\left(h_{0}-m_{1}\right)$ has a positive spectrum since it is essentially equivalent to the kinetic energy operator. One can then check that the above Bethe-Salpeter wave function has the correct spectral properties [7, 9]. For $x^{0}>0$, it has singularities for positive values of the relative energy variable, and for $x^{0}<0$, 
it has singularities for negative values of the relative energy variable. Furthermore, by decomposing $\Phi$ as

$$
\Phi=\theta\left(x^{0}\right) \Phi_{+}+\theta\left(-x^{0}\right) \Phi_{-},
$$

one verifies that the conjugate Bethe-Salpeter wave function $\bar{\Phi}$, identified from the comparison of Eqs. (18) and (20) with the use of Eq. (19), satisfies the correct definition (with the antichronological product):

$$
\bar{\Phi}=\theta\left(-x^{0}\right) \Phi_{+}^{\dagger} \gamma_{10}+\theta\left(x^{0}\right) \Phi_{-}^{\dagger} \gamma_{10} .
$$

The Bethe-Salpeter wave function could also have been constructed from the resolution of the equations it satisfies. By taking in Eq. (2) the limit $x_{2}^{\prime 0} \rightarrow-\infty$ and using the cluster decomposition (20), one finds the equations:

$$
\begin{aligned}
& \left(i \gamma_{1} \cdot \partial_{1}-m_{1}-\gamma_{10} V(r) \theta\left(x_{2}^{0}-x_{1}^{0}\right)\right) \Phi=0, \\
& i\left(\partial_{10}+\partial_{20}\right) \Phi=P_{0} \Phi
\end{aligned}
$$

the solution of which is precisely the function defined in Eqs. (23) and (26), when the continuity condition at $x^{0}=0$ is used. 


\section{Covariant gauges}

For completeness, we briefly sketch the derivation in the case of linear covariant gauges. Here, a complication arises because of the presence of gauge photons (scalar and longitudinal) which create in the spectrum of states additional cuts starting from the bound state poles [19]. These gauge dependent cuts are usually removed by appropriately associating, through Ward-Takahashi identities, with the exchanged photons the photons contributing to the radiative corrections and taking in the cluster decomposition formula (20) the limits $X^{0} \rightarrow \infty$ and $X^{\prime 0} \rightarrow-\infty$, while keeping $x^{0}$ and $x^{\prime 0}$ finite. With this procedure one selects the bound state spectrum [17], which is the object of interest. An illustration of this cancellation can be found in Ref. [12] for the static case. We shall not consider here the radiative corrections, which are more complicated than in the static limit, but shall indicate when necessary, their precise role.

In linear covariant gauges, characterized by a gauge parameter $\xi$, the photon propagator in momentum space is:

$$
D_{\mu \nu}(k)=-\left(g_{\mu \nu}-\xi \frac{k_{\mu} k_{\nu}}{k^{2}}\right) \frac{i}{k^{2}+i \epsilon} .
$$

Defining the functions

$$
\begin{aligned}
& \chi^{\prime}(x)=-\frac{i e^{2}}{4 \pi^{2}} \frac{1}{2|\mathbf{x}|} \ln \left(\frac{|\mathbf{x}|+\mathbf{x}^{\mathbf{0}}+\mathbf{i} \epsilon}{|\mathbf{x}|-\mathbf{x}^{\mathbf{0}}+\mathbf{i} \epsilon}\right), \\
& \Delta(x)=\int \frac{d^{4} k}{(2 \pi)^{4}} \frac{e^{-i k . x}}{\left(k^{2}+i \epsilon\right)^{2}}=\frac{i}{16 \pi^{2}} \ln \left(-\mu^{2} x^{2}\right),
\end{aligned}
$$

$\left[\chi^{\prime}\right.$ being the derivative, in the Feynman gauge, with respect to $x^{0}$, of the function $\chi$ defined in Ref. [12],] one finds for the effective potential $\widetilde{A}_{\mu}$ [Eq. (7)] the expression:

$$
e^{2} \widetilde{A}_{\mu}^{(\xi)}\left(x, x_{2}, x_{2}^{\prime}\right)=i g_{\mu 0}\left(\chi^{\prime}\left(x-x_{2}\right)-\chi^{\prime}\left(x-x_{2}^{\prime}\right)\right)-i e^{2} \xi \partial_{\mu}\left(\Delta\left(x-x_{2}\right)-\Delta\left(x-x_{2}^{\prime}\right)\right) .
$$

The fact that the $\xi$-dependent part od $\widetilde{A}_{\mu, \xi}$ is a derivative term, makes it possible its immediate elimination from Eqs. (2)-(3). One finds for $G^{(\xi)}$ the following $\xi$ dependence:

$$
G^{(\xi)}=\exp \left\{i e^{2} \xi\left(\Delta\left(x_{1}-x_{2}\right)-\Delta\left(x_{1}-x_{2}^{\prime}\right)-\Delta\left(x_{1}^{\prime}-x_{2}\right)+\Delta\left(x_{1}^{\prime}-x_{2}^{\prime}\right)\right)\right\} G^{(0)},
$$

which is precisely the general transformation law of the two-particle Green's function under covariant gauge transformations of the photon propagator (radiative corrections being neglected here) [20, 21, 22, 23]. It has been shown that these $\xi$-dependent gauge factors do not change the bound state pole positions of Green's functions [15, 16]. It is therefore sufficient to work in the Feynman gauge $(\xi=0)$, which is also similar to the scalar interaction case. (We shall henceforth omit the index 0 from the Green's function.) 
The function $\chi^{\prime}$ has the following asymptotic behavior:

$$
\chi^{\prime}(x) \underset{\left|x^{0}\right| \rightarrow \infty}{\longrightarrow} \epsilon\left(x^{0}\right) \frac{1}{2} V(|\mathbf{x}|)+\mathbf{O}\left(\frac{1}{\mathbf{x}^{\mathbf{0}}}\right) .
$$

It is the $O\left(1 / x^{0}\right)$ term which is gauge dependent (it is proportional to the derivative of the function $\Delta$ of Eq. (3) and vanishes in the gauge $\xi=-2$ ) and must be cancelled by a corresponding piece coming the radiative corrections [12]. We shall assume that this cancellation has occurred and thus the next-to-leading term in the asymptotic part of $\chi^{\prime}$ is $O\left(1 / x^{02}\right)$.

Replacing $\widetilde{A}_{\mu}^{(0)}$ by its expression (4) in Eqs. (2)-(3) and isolating again the asymptotic part of the potential, as in Eqs. (14)-(15), one obtains:

$$
\begin{aligned}
\left(i \gamma_{1} . \partial_{1}-\right. & \left.m_{1}-\gamma_{10} V(r)-\gamma_{10}\left[\left(\chi^{\prime}\left(x_{2}-x_{1}\right)-\frac{1}{2} V(r)\right)+\left(\chi^{\prime}\left(x_{1}-x_{2}^{\prime}\right)-\frac{1}{2} V(r)\right)\right]\right) G \\
=G_{20}\left(x_{2}^{\prime}-x_{2}\right) i \delta^{4}\left(x_{1}-x_{1}^{\prime}\right) &
\end{aligned}
$$

and a similar conjugate equation. The remaining part of the resolution of these equations parallels that utilized in the Coulomb gauge. One arrives at an expression analogous to that of Eq. (16), where the potential part $V(1-\theta \theta)$ is replaced by the bracket term of Eq. (7) (with $x_{1}^{0}$ replaced by the integration variable $y_{1}^{0}$ ). One then takes the limits $x_{2}^{0}, x_{1}^{0} \rightarrow \infty$ and $x_{2}^{\prime 0}, x_{1}^{\prime 0} \rightarrow-\infty$, by keeping $x^{0}$ and $x^{\prime 0}$ finite. Because the difference $\chi^{\prime}(x)-V / 2$ behaves as $O\left(1 / x^{02}\right)$, in the part containing $\chi^{\prime}\left(x_{2}-y_{1}\right)-V / 2$ it is the values of $y_{1}^{0}$ close to $x_{2}^{0}$ that contribute, while in the part containing $\chi^{\prime}\left(y_{1}-x_{2}^{\prime}\right)-V / 2$ it is the values of $y_{1}^{0}$ close to $x_{2}^{\prime 0}$ that are relevant. Thus these two parts receive contributions from disjoint intervals and the $T$-product factorizes again [Eq. (17)], with the first integral lying from $-\infty$ to $x_{1}^{0}$ and the second integral from $x_{1}^{\prime 0}$ to $\infty$. One ends up, for the bound state spectrum, with the same conclusion as in the Coulomb gauge. 


\section{References}

[1] S. J. Brodsky, in Brandeis Lectures 1969, eds. M. Chrétien and E. Lipworth (Gordon and Breach, New York, 1971), p. 93.

[2] W. Dietrich, Phys. Rev. D 1 (1970) 3345.

[3] A. R. Neghabian and W. Glöckle, Can. J. Phys. 61 (1983) 85.

[4] F. Gross, Phys. Rev. 186 (1969) 1448.

[5] C. Fronsdal and R. W. Huff, Phys. Rev. D 3 (1971) 933.

[6] J. Mourad and H. Sazdjian, J. Math. Phys. 29 (1994) 6379.

[7] G.C. Wick, Phys. Rev. 96 (1954) 1124.

[8] R.E. Cutkosky, Phys. Rev. 96 (1954) 1135.

[9] N. Nakanishi, Suppl. Prog. Theor. Phys. 43 (1969) 1.

[10] N. Nakanishi, Prog. Theor. Phys. Suppl. 95 (1988) 1; in Extented Objects and Bound Systems, proceedings of the Karuizawa International Symposium, 1992, eds. O. Hara, S. Ishida and S. Naka (World Scientific, Singapore, 1992), p. 109.

[11] Y. Ohnuki, Y. Takao and H. Umezawa, Prog. Theor. Phys. 23 (1960) 273.

[12] H. Jallouli and H. Sazdjian, J. Phys. G: Nucl. Part. Phys. 22 (1996) 1119.

[13] F.L. Feinberg, Phys. Rev. D 17 (1978) 2659;

W.E. Caswell and G.P. Lepage, Phys. Lett. B 167 (1986) 437;

H. Georgi, Phys. Lett. B 240 (1990) 447;

F. Hussain and G. Thompson, ICTP preprint IC/95/15, hep-ph/9502241.

[14] J. G. Körner and G. Thompson, Phys. Lett. B 264 (1991) 185.

[15] G. T. Bodwin and D. R. Yennie, Phys. Rep. C 43 (1978) 413.

[16] G. Feldman, T. Fulton and D.L. Heckathorn, Nucl. Phys. B167 (1980) 364; B174 (1980) 89.

[17] M. Gell-Mann and F. Low, Phys. Rev. 84 (1951) 350.

[18] R. P. Feynman, Phys. Rev. 84 (1951) 108.

[19] K. Johnson and B. Zumino, Phys. Rev. Lett. 3 (1959) 351. 
[20] L. D. Landau and I. M. Khalatnikov, Sov. Phys. JETP 2 (1956) 69.

[21] B. Zumino, J. Math. Phys. 1 (1959) 1.

[22] S. Okubo, Nuovo Cimento 15 (1960) 949.

[23] R. Barbieri, M. Ciafaloni and P. Menotti, Nuovo Cimento 55A (1968) 701. 